\title{
La colección de Entomología Acuática del Museo de Zoología, Universidad de Costa Rica: lista actualizada de géneros, importancia y retos futuros
}

\section{Monika Springer}

Escuela de Biología, Museo de Zoología y Centro de Investigación en Ciencias del Mar y Limnología (CIMAR), Universidad de Costa Rica, San Pedro de Montes de Oca, 2060 San José, Costa Rica; monika.springer@ucr.ac.cr

\author{
Recibido 10-X-2018. Corregido 15-II-2019. Aceptado 26-III-2019.
}

\begin{abstract}
The aquatic entomology collection in the Zoological Museum at the University of Costa Rica: updated checklist of genera, importance, and future challenges. Aquatic macroinvertebrates, and especially insects, are the group of organisms most commonly used to determine the health of ecosystems in water quality studies and freshwater biomonitoring. The most important scientific collection of this group in Costa Rica is located in the Zoological Museum of the University of Costa Rica (MZUCR) and includes material from scientific research, thesis and student projects, as well as samples from environmental studies and aquatic monitoring by consultants and institutions. In this work, an updated list of the collection's material is presented, which includes 98 families and 341 identified taxa (genera, tribes or subfamilies) from eleven insect orders, with Coleoptera and Diptera as the most taxonomical diverse orders. Studies which resulted from revisions of the collection material are cited, including new species, genera, and family records, as well as descriptions of new species and associations of immature stages with their adults. The main challenges for this collection include the taxonomic identification of certain groups, keeping up with the constant collection growth and updating the associated database.
\end{abstract}

Key words: freshwater; aquatic insects; macroinvertebrates; taxonomy; inventory.

Springer, M. (2019). La colección de Entomología Acuática del Museo de Zoología, Universidad de Costa Rica: lista actualizada de géneros, importancia y retos futuros. Revista de Biología Tropical, 67(2) Suplemento, S200-S211.

La importancia de las colecciones científicas y su aporte al conocimiento y a la conservación de la biodiversidad, han sido enfatizados por muchos autores (e.g. Lane, 1996; Pinto et al., 2010; Pyke \& Ehrlich, 2010; Drew, 2011; Hoeksema et al., 2011; Kress, 2014). Las investigaciones que se realizan con base en los especímenes depositados, y la información asociada a éstos, incluyen estudios taxonómicos, sistemáticos, morfológicos, genéticos, patológicos, ecológicos y biogeográficos. Además, pueden revelar información importante para entender los factores que amenazan a muchas especies y permiten analizar vacíos de conservación. Las colecciones científicas proveen beneficios financieros directos a la sociedad, por reducir los costos de muchas investigaciones, incluyendo estudios sobre vectores de enfermedades, invasiones biológicas y cambio climático (Suarez \& Tsutsui, 2004).

En Costa Rica, las colecciones zoológicas más importantes están albergadas en el Museo Nacional y en el Museo de Zoología de la Universidad de Costa Rica (MZUCR). Este último fue fundado en 1966 y contiene en la actualidad más de cinco millones de especímenes de la gran mayoría de los grupos de vertebrados e invertebrados terrestres, marinos y de agua dulce, entre los cuales se encuentran los insectos acuáticos. La colección 
de Entomología Acuática del MZUCR fue establecida en 1992 y el primer listado publicado incluyó 278 géneros de 92 familias y 11 órdenes de insectos (Springer, 1998). Además de los insectos, esta colección incluye otros grupos de macroinvertebrados acuáticos, como moluscos, crustáceos, ácaros, gusanos planos y sanguijuelas, entre otros.

Los macroinvertebrados acuáticos y en particular los insectos, son de especial relevancia para la conservación de los ambientes de aguas continentales, ya que son considerados excelentes indicadores que reflejan de manera confiable la condición de los ambientes dulceacuícolas y la calidad de sus aguas (Rosenberg \& Resh, 1993; Bonada, Prat, Resh, \& Statzner, 2006; Springer, 2010). Por esta razón, durante las últimas dos décadas, el interés por estudiar este grupo ha aumentado notablemente en el país (Springer, 2008; Springer, Echeverría, \& Gutiérrez, 2014) y actualmente son utilizados con mucha frecuencia en estudios ambientales, evaluaciones de calidad de agua y monitoreos periódicos, especialmente de ríos y quebradas. Como consecuencia, el ingreso de muestras a la colección del MZUCR ha aumentado en forma exponencial durante las últimas dos décadas.

Este importante crecimiento de la colección ha generado a su vez un mayor conocimiento taxonómico sobre la entomofauna acuática del país. Por lo tanto, el objetivo de este trabajo consiste en presentar un listado actualizado de los géneros de insectos acuáticos depositados en la Colección de Entomología Acuática del Museo de Zoología de la Universidad de Costa Rica, así como evidenciar su importancia por medio de las publicaciones generadas a partir de las muestras depositadas en esta colección.

\section{MATERIALES Y MÉTODOS}

El material depositado en la colección ha sido recolectado por muchas personas de distintas instituciones a través de diversos proyectos de investigación, trabajos de campo para cursos y tesis de estudiantes, así como estudios y monitoreos ambientales. Los métodos de recolecta implementados varían según el tipo de estudio y hábitat, e incluyen métodos cualitativos (recolecta directa con pinzas y redes de mano) y cuantitativos (e.g. red surber, sustrato artificial), con redes de luz de malla desde las $200 \mu \mathrm{m}$ hasta $1 \mathrm{~mm}$. Los ambientes acuáticos representados incluyen tanto ambientes lóticos (ríos, arroyos, canales), como lénticos (lagos, lagunas, embalses, charcos, turberas, pantanos), además de otros ambientes como fitotelmatas (agua acumulada en estructuras de plantas, como tanques de bromelias) y hábitats artificiales (llantas, piletas, entre otras). Los sitios de recolecta abarcan las diferentes ecorregiones en ambas vertientes del país, desde el nivel del mar hasta los 3600 m.s.n.m. Adicionalmente, también existen muestras de otros países en la colección, aunque el presente trabajo se enfoca únicamente en el material recolectado en Costa Rica.

La gran mayoría de los especímenes están preservados en alcohol al 70 o $95 \%$ (según tipo de estudio) por lo que se encuentran ubicados en la colección húmeda. Por otro lado, los adultos voladores de ciertos grupos, como por ejemplo Odonata, Trichoptera y Megaloptera, se preservan en seco (guardados en sobres transparentes o montados en alfileres, según el grupo) o montados en láminas fijas (algunas familias de Diptera). La totalidad de las muestras se encuentran depositadas en el Museo de Zoología de la Universidad de Costa Rica (MZUCR), ubicado en la Escuela de Biología en la sede Rodrigo Facio en San Pedro de Montes de Oca.

La colección húmeda de Entomología Acuática está organizada en dos secciones: la sección taxonómica y la geográfica. En la sección taxonómica, las muestras se encuentran organizadas por órdenes, familias y géneros, y en algunos grupos por especie (e.g. Hemiptera, Plecoptera). La totalidad de las muestras ubicadas en esta sección está incluida en la base de datos. Por otro lado, en la sección geográfica se ubican las muestras generadas por proyectos de investigación, tesis y proyectos de estudiantes, así como estudios y monitoreos ambientales. Estos provienen de diversos centros de investigación y universidades, consultores e 
instituciones gubernamentales, como el Instituto Costarricense de Electricidad (ICE) o la Compañía Nacional de Fuerza y Luz (CNFL), así como del Plan Nacional de Monitoreo de los Cuerpos de Agua Superficiales, coordinado por la Dirección de Aguas, del Ministerio de Ambiente y Energía. En esta sección de la colección, cada muestra agrupa los taxones recolectados durante un evento de muestreo (una misma fecha y un mismo sitio). Esta sección representa la mayor parte de la colección y debido al gran volumen de muestras que ingresan constantemente, aún se está en proceso de digitalizar la información asociada.

La base de datos de la colección cuenta actualmente con cerca de 12000 registros, aunque se estima que pueda llegar a superar los 60000 registros, una vez que se logre incluir la totalidad de las muestras depositadas en la sección geográfica. La base de datos incluye la información asociada a la recolecta, así como la identificación taxonómica y la ubicación de la muestra en la colección. Un registro corresponde a todos los individuos de un taxón específico (especie o género), recolectados en un mismo sitio y una fecha dada.

Para garantizar la correcta identificación de los organismos, especialmente aquellos depositados en la sección taxonómica, se cuenta con el apoyo de especialistas taxónomos en diferentes grupos. En aquellos casos donde, por diversas razones, aún no ha sido posible la identificación del género, se utiliza "Gen. indet."; además, especialmente en el caso de algunos coleópteros y dípteros, se indica la subfamilia o el tribu, al cual se logró identificar. La abreviación "cf." ("confer") antes de un género se utiliza para aquellos casos donde el organismo correspondiente calza con la descripción del género señalado, aunque no se puede asegurar su identificación por la carencia de claves taxonómicas y la falta de descripciones de otros géneros de la misma familia.

Dado que la colección está enfocada en los estadíos acuáticos de cada taxón (ubicados en la colección húmeda), en los siguientes órdenes se cuenta mayoritariamente con estadíos inmaduros (ninfas o larvas): Ephemeroptera,
Odonata, Plecoptera, Blattodea, Trichoptera, Lepidoptera, Neuroptera, Megaloptera y Diptera. En el caso del orden Hemiptera se incluyen tanto ninfas como adultos de las familias acuáticas, mientras que para Coleoptera la colección puede incluir, dependiendo de cada familia, solo larvas, solo adultos, o larvas y adultos de cada taxón (indicado en el listado después del nombre de cada familia). Para el orden Odonata, la lista presentada incluye además los registros basados en los adultos, ubicados en la colección seca, lo cual también es indicado en la lista.

\section{RESULTADOS}

Listado de géneros depositados: En el cuadro 1 se presenta el listado de las familias y de los géneros depositados en la colección para cada uno de los 11 órdenes. En total se encuentran 98 familias y 341 géneros identificados, mientras que al menos otros 51 géneros están aún sin identificar o únicamente cuentan con la identificación a nivel de tribu o subfamilia. El orden con la mayor diversidad taxonómica en la colección es Coleoptera, con 20 familias y 86 géneros identificados, además de otros 15 sin identificar. Del orden Diptera se incluyen representantes de 19 familias, con únicamente 45 géneros identificados, aunque muchos de éstos aún no se pueden confirmar con certeza (indicados con "cf." en la lista). Este orden contiene además la mayor cantidad de organismos sin clasificar, con larvas de al menos una familia y 23 géneros aún por identificar, aunque algunos sí se han podido identificar al menos a subfamilia o tribu. Otros órdenes con alta diversidad taxonómica son Odonata (14 familias, 65 géneros), Hemiptera (15 familias, 45 géneros), Trichoptera (15 familias, 44 géneros) y Ephemeroptera (9 familias, 44 géneros). Estos órdenes cuentan con la gran mayoría del material identificado a nivel de género y solamente en pocas familias aún quedan algunos sin identificar.

Con respecto a la primera lista publicada (Springer, 1998), la colección ha aumentado en seis familias y 99 géneros identificados, además 
CUADRO 1

Lista de órdenes, familias y géneros de insectos acuáticos depositados en la colección de Entomología Acuática del Museo de Zoología de la Universidad de Costa Rica (MZUCR)

TABLE 1

List of orders, families and genera of aquatic insects, deposited in

the Aquatic Entomology Collection at the Museum of Zoology, University of Costa Rica (MZUCR)

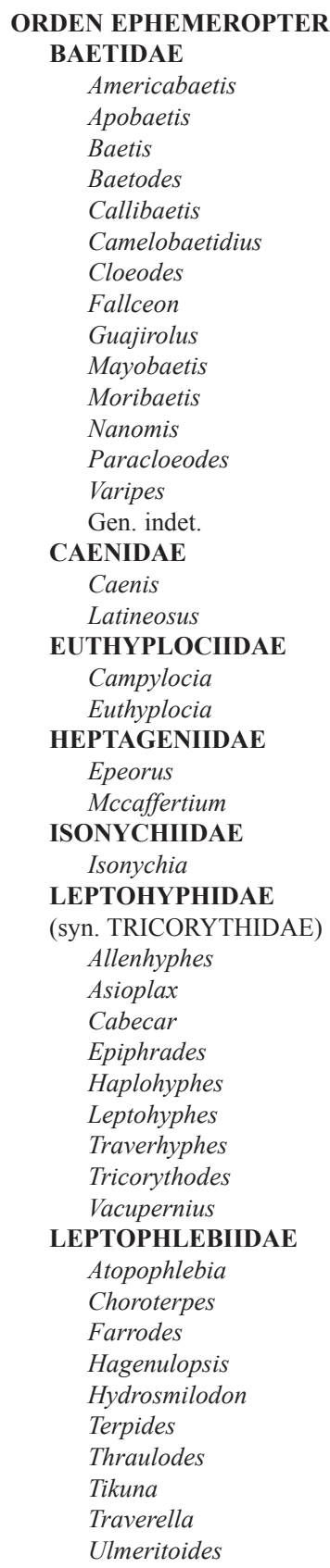

\section{OLIGONEURIIDAE \\ Lachlania \\ POLYMITARCYIDAE \\ Campsurus \\ Tortopsis \\ Tortopus}

ORDEN ODONATA (larvas y adultos; *solo adultos)

SUBORDEN ANISOPTERA

AESHNIDAE

Aeshna

Anax

Coryphaeschna

Gynacantha

Remartinia

Rhioaeschna*

Triacanthagyna

Gen. indet.

CORDULEGASTRIDAE

Cordulegaster

CORDULIIDAE

Neocordulia

LIBELLULIDAE

Anatya*

Brachymesia

Brechmorhoga

Cannaphila

Dythemis

Elasmothemis

Erythemis

Erythrodiplax

Libellula

Macrothemis

Miathyria

Micrathyria

Nephepeltia

Orthemis

Paltothemis

Pantala

Perithemis

Pseudoleon

Rhodopygia

Sympetrum

Tramea

Uracis*

Zenithoptera*

Gen indet.

Ulmeritoides 


\begin{tabular}{|c|c|}
\hline GOMPHIDAE & ORDEN BLATTARIA \\
\hline Agriogomphus & BLABERIDAE (ninfas) \\
\hline Aphylla & Epilampra \\
\hline Desmogomphus & Gen. indet. \\
\hline \multicolumn{2}{|l|}{ Epigomphus } \\
\hline Erpetogomphus & ORDEN HEMIPTERA (ninfas \& adultos) \\
\hline Perigomphus & INFRAORDEN NEPOMORPHA \\
\hline Phyllocycla & BELOSTOMATIDAE \\
\hline Phyllogomphoides & Abedus \\
\hline Progomphus & Belostoma \\
\hline SUBORDEN ZYGOPTERA & Lethocerus \\
\hline CALOPTERYGIDAE & CORIXIDAE \\
\hline Hetaerina & Centrocorisa \\
\hline COENAGRIONIDAE & Graptocorixa \\
\hline (incluye lo que era antes Protoneuridae y & Tenegobia \\
\hline Pseudostigmatidae) & Trichocorixa \\
\hline Acanthagrion & Gen. indet. \\
\hline Anisagrion & GELASTOCORIDAE \\
\hline Argia & Gelastocoris \\
\hline Enallagma & Nerthra \\
\hline Ischnura & NAUCORIDAE \\
\hline Leptobasis & Ambrysus \\
\hline Mecistogaster & Cryphocricos \\
\hline Megaloprepus & Ctenipocoris \\
\hline Metaleptobasis* & Limnocoris \\
\hline Nehalennia & Pelocoris \\
\hline Neoerythromma & NEPIDAE \\
\hline Neoneura & Curicta \\
\hline Protoneura & Ranatra \\
\hline Psaironeura & NOTONECTIDAE \\
\hline Telebasis & Buепоа \\
\hline Gen. indet. & Martarega \\
\hline LESTIDAE & Notonecta \\
\hline Archilestes & OCHTERIDAE \\
\hline Lestes & Ochterus \\
\hline HETERAGRIONIDAE & PLEIDAE \\
\hline (antes MEGAPODAGRIONIDAE) & Neoplea \\
\hline Heteragrion & Paraplea \\
\hline PHILOGENIDAE & POTAMOCORIDAE \\
\hline (antes MEGAPODAGRIONIDAE) & Potamocoris \\
\hline Philogenia & INFRAORDEN GERROMORPHA \\
\hline THAUMATONEURIDAE & GERRIDAE \\
\hline (antes MEGAPODAGRIONIDAE) & Brachymetra \\
\hline Thaumatoneura & Eurygerris \\
\hline PERILESTIDAE & Halobates \\
\hline Perissolestes & Limnogonus \\
\hline PLATYSTICTIDAE & Metrobates \\
\hline Palaemnema & Neogerris \\
\hline POLYTHORIDAE & Platygerris \\
\hline Cora & Potamobates \\
\hline Miocora* & Rheumatobates \\
\hline & Tachygerris \\
\hline ORDEN PLECOPTERA & Telmatometra \\
\hline PERLIDAE (ninfas) & Telmatometroides \\
\hline Anacroneuria & Trepobates \\
\hline Perlesta & HYDROMETRIDAE \\
\hline & Hydrometra \\
\hline
\end{tabular}




\begin{tabular}{|c|c|}
\hline HEBRIDAE & ELMIDAE (larvas \& adultos) \\
\hline Hebrus & Austrolimnius \\
\hline Lipogomphus & Cylloepus \\
\hline MESOVELIIDAE & Disersus \\
\hline Mesovelia & Heterelmis \\
\hline Mesoveloidea & Hexacylloepus \\
\hline VELIIDAE & Hexanchorus \\
\hline Microvelia & Macrelmis \\
\hline Platyvelia & Microcylloepus \\
\hline Rhagovelia & Neocylloepus \\
\hline Stridulivelia & Neoelmis \\
\hline INFRAORDEN LEPTOPODOMORPHA & Notelmis \\
\hline SALDIDAE & Onychelmis \\
\hline \multirow[t]{2}{*}{ Gen. indet. } & Phanocerus \\
\hline & Pharceonus \\
\hline ORDEN MEGALOPTERA & Pseudodisersus \\
\hline CORYDALIDAE (larvas) & Stenhelmoides \\
\hline Corydalus & Xenelmis \\
\hline Chloronia & Gen. indet. \\
\hline Platyneuromus & GYRINIDAE (larvas \& adultos) \\
\hline SIALIDAE (larvas) & Dineutus \\
\hline \multirow[t]{2}{*}{ Sialis } & Enhydrus \\
\hline & Gyretes \\
\hline ORDEN NEUROPTERA & Gyrinus \\
\hline SISYRIDAE (larvas) & HYDRAENIDAE (adultos) \\
\hline \multirow[t]{2}{*}{ Sisyra } & Hydraena \\
\hline & Ochthebius \\
\hline ORDEN COLEOPTERA & HALIPLIDAE (larvas) \\
\hline CHRYSOMELIDAE (larvas) & Haliplus \\
\hline Cephalocela & HYDROPHILIDAE (larvas \& adultos) \\
\hline Gen. indet. & Anacaena \\
\hline CURCULIONIDAE (larvas \& adultos) & Berosus \\
\hline Lissorhoptrus & Derallus \\
\hline Gen. indet. & Enochrus \\
\hline DYTISCIDAE (larvas \& adultos) & Helochares \\
\hline Agametrus & Hemiosus \\
\hline Anodocheilus & Hydraena \\
\hline Celina & Hydrochus \\
\hline Copelatus & Hydrophilus \\
\hline Cybister & Hydrous \\
\hline Derovatellus & Paracymus \\
\hline Desmopachria & Phaenonotum \\
\hline Hydraticus & Tropisternus \\
\hline Hydrovatus & Gen. indet. \\
\hline Laccodytes & Sphaeridiinae Gen. indet. \\
\hline Laccophilus & HYDROSCAPHIDAE (larvas \& adultos) \\
\hline Liodessus & cf. Yara \\
\hline Megadytes & LAMPYRIDAE (larvas) \\
\hline Neobidessus & Gen. indet. \\
\hline Neoclypeodytes & LIMNICHIDAE (adultos) \\
\hline Pachydrus & Limnochoderus \\
\hline Rhantus & Gen. indet. \\
\hline Thermonectes & LUTROCHIDAE (larvas \& adultos) \\
\hline Gen. indet. & Lutrochus \\
\hline DRYOPIDAE (adultos) & NOTERIDAE (larvas \& adultos) \\
\hline Dryops & Hydrocanthus \\
\hline Elmoparnus & Mesonoterus \\
\hline Helichus & Suphis \\
\hline Pelonomus & \\
\hline
\end{tabular}




\begin{tabular}{|c|c|}
\hline Suphisellus & HYDROPTILIDAE \\
\hline Gen. indet. & Alisotrichia \\
\hline PSEPHENIDAE (larvas \& adultos) & Anchitrichia \\
\hline Neoeubria & Brysopteryx \\
\hline Psephenops & Hydroptila \\
\hline Psephenus & Leucotrichia \\
\hline Eubriinae Gen. indet. & Mayatrichia \\
\hline PTILIIDAE (adultos) & Metrichia \\
\hline Gen. indet & Neotrichia \\
\hline PTILODACTYLIDAE (larvas) & Ochrotrichia \\
\hline Anchytarsus & Oxyethira \\
\hline SCIRTIDAE (larvas) & Rhyacopsyche \\
\hline Gen. indet. & Zumatrichia \\
\hline SPHAERIUSIDAE (adultos) & Gen. indet. \\
\hline Sphaerius & Leucotrichini Gen. indet. \\
\hline STAPHYLINIDAE (larvas \& adultos) & LEPIDOSTOMATIDAE \\
\hline Atanygnathus & Lepidostoma \\
\hline Bamona & LEPTOCERIDAE \\
\hline Carpelimus & Atanatolica \\
\hline $\begin{array}{l}\text { Carpeltmus } \\
\text { Coproporus }\end{array}$ & Nectopsyche \\
\hline $\begin{array}{l}\text { Coproporus } \\
\text { Diestota }\end{array}$ & Oecetis \\
\hline $\begin{array}{l}\text { Diestota } \\
\text { Evaesthetus }\end{array}$ & Triaenodes \\
\hline & Triplectides \\
\hline $\begin{array}{l}\text { Homaeotarsus } \\
\text { Myllaena }\end{array}$ & LIMNEPHILIDAE \\
\hline Myllaena & Limnephilus \\
\hline Ocacea & ODONTOCERIDAE \\
\hline Stenus & Marilia \\
\hline Thinodromus & PHILOPOTAMIDAE \\
\hline Tinobius & Chimarra \\
\hline Trogactus & Wormaldia \\
\hline Aleocharinae Gen. indet. & Gen. indet. (Chimarrhodella?) \\
\hline Oxytelinae Gen.indet. & POLYCENTROPODIDAE \\
\hline Staphylininae Gen. indet. & Cernotina \\
\hline & Cyrnellus \\
\hline ORDEN TRICHOPTERA (larvas \& pupas) & Polycentropus \\
\hline ANOMALOPSYCHIDAE & Polyplectropus \\
\hline Contulma & XIPHOCENTRONIDAE \\
\hline CALAMOCERATIDAE & Xiphocentron \\
\hline Banyallarga & Gen. indet. \\
\hline \multicolumn{2}{|l|}{ Phylloicus } \\
\hline ECNOMIDAE & ORDEN LEPIDOPTERA \\
\hline Austrotinodes & CRAMBIDAE (larvas \& pupas) \\
\hline GLOSSOSOMATIDAE & Argyractis \\
\hline Culoptila & Neargyractis \\
\hline Mortionella & Paraponyx \\
\hline Protoptila & Petrophila \\
\hline HELICOPSYCHIDAE & Gen. indet. \\
\hline \multicolumn{2}{|l|}{ Helicopsyche } \\
\hline HYDROBIOSIDAE & ORDEN DIPTERA (larvas \& pupas) \\
\hline Atopsyche & SUBORDEN NEMATOCERA \\
\hline HYDROPSYCHIDAE & BLEPHARICERIDAE \\
\hline Calosopsyche & Paltostoma \\
\hline Centromacronema & CERATOPOGONIDAE \\
\hline Leptonema & cf. Alluaudomyia \\
\hline Macronema & cf. Atrichopogon \\
\hline Macrostemum & cf. Forcipomyia \\
\hline Plectromacronema & cf. Probezzia \\
\hline Plectropsyche & cf. Stilobezzia \\
\hline \multirow{2}{*}{$\begin{array}{l}\text { Plectropsyche } \\
\text { Smicridea }\end{array}$} & Ceratopogoninae Gen. indet. \\
\hline & Forcipomyinae Gen. indet. \\
\hline
\end{tabular}




\begin{tabular}{lc}
\hline CHAOBORIDAE & TIPULIDAE \\
Chaoborus & Hexatoma \\
CHIRONOMIDAE & Limonia \\
Chironomus & Molophilus \\
Rheotanytarsus & Tipula \\
Stenochironomus & Limoniinae Gen. indet. \\
Xestochironomus & Tipulinae Gen. indet. \\
Chironominae Gen. indet. & SUBORDEN BRACHYCERA - ORTHORRHAPHA \\
Chironimini Gen. indet. & ATHERICIDAE \\
Orthocladiinae Gen. indet. & Suragina \\
Pentaneurini Gen. indet. & DOLICHOPODIDAE \\
Podonominae Gen. indet. & Gen. indet. \\
Tanypodini Gen. indet. & EMPIDIDAE \\
Tanytarsini Gen. indet. & Neoplasta \\
CORETHRELLIDAE & Hemerodromia \\
Corethrella & Gen. indet. \\
CULICIDAE & STRATIOMYIDAE \\
Aedes & Caloparyphus \\
Aedeomyia & Euparyphus \\
Anopheles & Myxosargus \\
Culex & Nemotelus \\
Haemogogus & Odontomyia \\
Mansonia & Stratiomys \\
Orthopodomyia & Gen. indet. \\
Psorophora & TABANIDAE \\
Toxorhynchites & cf. Chrysops \\
Wyeomyia & cf. Tabanus \\
Gen. indet. & Gen. indet. \\
DIXIDAE & SUBORDEN BRACHYCERA - CYCLORRHAPHA \\
Dixella & EPHYDRIDAE \\
Gen. indet. (cf. Meringodixa) & Gen. indet. \\
PSYCHODIDAE & MUSCIDAE \\
Clognia & cf. Lispe \\
Maruina & cf. Limnophora \\
Pericoma & Gen. indet. \\
Gen. indet. & SCIOMYZIDAE \\
SIMULIIDAE & Gen. indet. \\
Gigantodax & SYRPIDAE \\
Simulium & Gen. indet. \\
& FAM. INDET. \\
& Gen. indet. \\
\hline & \\
& \\
& \\
&
\end{tabular}

de otros 13 géneros sin identificar (algunos de estos identificados a nivel de tribu o subfamilia). Las familias que se han podido incluir después de la primera publicación son: Potamocoridae e Hydrometridae del orden Hemiptera, Ecnomidae de Trichoptera e Hyraenidae, Sphaeriusidae y Ptiliidae del orden Coleoptera. Estas familias representan organismos de distribución restringida que se han recolectado en únicamente pocos sitios (e.g. Ecnomidae), o bien organismos de tamaño muy pequeño por lo que ha sido difícil su recolecta (e.g. Sphaeriusidae). Lo mismo ocurre para muchos de los géneros que se han podido registrar posterior a la primera lista publicada, aunque en este caso el aumento se puede contribuir en gran parte también a una mayor capacidad taxonómica. Esto ha sido posible gracias al apoyo de muchos taxónomos de diversas instituciones y países, quienes han colaborado con la identificación del material, la elaboración de claves taxonómicas y han ayudado en la capacitación 
de estudiantes quienes han realizado sus tesis en algún grupo específico (e.g. Hemiptera, Plecoptera y Odonata). Este hecho se evidencia además en las publicaciones generadas, las cuales se detallan a continuación.

Aportes al conocimiento taxonómico: $\mathrm{La}$ revisión de las muestras depositadas en la colección ha resultado en el descubrimiento y la descripción de nuevas especies, especialmente en el orden Hemiptera (Herrera, 2013a,b; Figueiredo Moreira, Pacheco-Chaves, Springer, \& Da Rocha Silva Cordeiro, 2015; Pacheco-Chaves, DaRocha Silva Cordeiro, Figueiredo Moreira, \& Springer, 2018). Además, ha revelado nuevos registros de especies para el país (Padilla-Gil \& Pacheco-Chaves, 2012; Herrera \& Springer, 2014; Pacheco-Chaves, Figueiredo Moreira, \& Springer, 2014; Figueiredo Moreira et al., 2015) y también para Centroamérica (Gutiérrez-Fonseca \& Springer, 2011; Herrera, 2013b). A partir del material depositado, se reportó por primera vez la presencia de la familia Potamocoridae (Hemiptera: Herrera \& Springer, 2012) y de los géneros Ctenipocoris (Hemiptera, Naucoridae: Herrera, 2013b), Perlesta (Plecoptera, Perlidae: Gutiérrez \& Springer, 2011) y Pharceonus (Coleoptera, Elmidae: Springer \& Acosta, 2003). Varios de estos primeros registros constituyeron además ampliaciones del rango de distribución para el respectivo taxón.

Adicionalmente, el material de la colección ha sido un importante insumo para la asociación y descripción de estadíos inmaduros de Plecoptera (Gutiérrez-Fonseca \& Springer, 2011), Odonata (Román-Heracleo, Springer, \& Ramírez, 2018; Ramírez, Altamiranda-Saavedra, Gutiérrez-Fonseca, \& Springer, 2011), Trichoptera (Springer \& Bermúdez, 2018) y Coleoptera (Springer \& Acosta, 2003); así como de las formas macrópteras del chinche naucórido Cryphocricos latus (Herrera \& González, 2014). La elaboración de claves de identificación únicamente ha sido posible a partir de la revisión de los organismos depositados, por ejemplo para los órdenes Ephemeroptera (Flowers, 2010), Odonata (Ramírez, 2010),
Plecoptera (Gutiérrez-Fonseca, 2010) y Trichoptera (Springer, 2006; 2010).

Finalmente, los datos asociados a los sitios de recolecta de cada muestra se han podido aprovechar para realizar mapas de distribución para cada especie en el país (e.g. GutiérrezFonseca \& Springer, 2011; Pacheco et al., 2014; Figueiredo Moreira et al., 2015) y analizar distribuciones altitudinales (e.g. GutiérrezFonseca \& Springer, 2015).

\section{DISCUSIÓN}

La sección de Entomología Acuática del Museo de Zoología de la Universidad de Costa Rica alberga sin duda la colección más grande y representativa de este grupo de organismos en Costa Rica. Hay varios grupos muy bien representados, con respecto al total de taxones reportados para el país, especialmente Ephemeroptera, Plecoptera, Odonata, Hemiptera, Megaloptera y Trichoptera. Aunque actualmente Coleoptera es el orden de mayor cantidad de géneros, aún contiene bastante material que no se ha podido identificar a un mayor nivel taxonómico, especialmente de las familias Hydrophilidae y Dytiscidae, las cuales cuentan con más de 30 géneros reportados para Costa Rica cada una.

El orden con la mayor diversidad y a la vez el menos conocido, especialmente a nivel de sus estadios inmaduros, es Diptera, con más del $95 \%$ de sus estadíos inmaduros sin describir (Brown et al., 2009). De este orden, únicamente algunas familias pequeñas y aquellas de importancia médica, como Culicidae y Simuliidae, cuentan con sus larvas y pupas debidamente descritas y claves taxonómicas para su identificación (e.g. Chaverri, 2009; Adler \& Currie, 2009). Hay algunas familias, como Tipulidae, Ceratopogonidae y Chironomidae, de las cuales se estima que puedan existir más de 1000 especies para cada una en el país (Brown et al., 2009). En estas familias, el conocimiento de las larvas es muy escaso y para poder generar claves taxonómicas para su debida identificación a nivel de género es necesario realizar asociaciones larva-pupa-adulto. 
Recientemente, se han realizado esfuerzos importantes para lograr estas asociaciones en la familia Chironomidae y así avanzar en el conocimiento de esta familia en Costa Rica (de la Rosa, 2015). Sin duda, el orden Diptera representa el mayor reto a nivel taxonómico, lo cual también queda evidenciado en la lista del presente trabajo, que incluye una gran cantidad de géneros sin identificar.

Aparte de los retos de una adecuada identificación taxonómica, la colección de Entomología Acuática del Museo de Zoología enfrenta también otros desafíos, entre los cuales se encuentran la eficiente incorporación de la gran cantidad de muestras, especialmente las generadas por los diversos programas de monitoreo y estudios ambientales, y la constante actualización de la base de datos. Actualmente, se realizan gestiones para poder migrar la base de datos actual al programa para manejo de colecciones biológicas, Specify 7 (Specify Collections Consortium, 2018). Esta nueva base de datos también permitirá un acceso más eficiente por medio de un portal en Internet, lo cual presentará un recurso muy valioso para los diferentes sectores involucrados en los estudios y la gestión de los ambientes acuáticos.

Indudablemente, la colección junto con la información asociada a cada muestra, constituyen una herramienta de apoyo importante, no solo para verificar identificaciones y desarrollar claves taxonómicas, sino también para generar nuevos registros para el país y la región, así como descubrir especies nuevas para la ciencia. Además, provee información adicional sobre la biología, historia natural, ecología y distribución de las familias, los géneros y las especies de los diferentes grupos de insectos acuáticos. Esta información es de interés no solo para estudiantes e investigadores quienes trabajan con fauna acuática, si no, además podrá ser de gran utilidad para tomadores de decisión, consultores y organizaciones no-gubernamentales que trabajan con el recurso hídrico.

Declaración de ética: la autora declara que está de acuerdo con esta publicación; que no existe conflicto de interés de ningún tipo; y que ha cumplido con todos los requisitos y procedimientos éticos y legales pertinentes. Todas las fuentes de financiamiento se detallan plena y claramente en la sección de agradecimientos. El respectivo documento legal firmado se encuentra en los archivos de la revista.

\section{AGRADECIMIENTOS}

Este trabajo fue posible gracias al aporte de muchas personas, quienes han depositado sus muestras en la colección. El apoyo de los distintos especialistas taxónomos ha sido crucial para corroborar, completar o corregir, cuando sea necesario, las identificaciones. Asimismo, la ayuda de muchos estudiantes ha sido crucial para garantizar un adecuado mantenimiento, ordenamiento y crecimiento de la colección. El SINAC, MINAE, ha sido la entidad que ha proporcionado los permisos de recolecta científica en sus diferentes áreas de conservación a través de los años y además una gran cantidad de personas ha colaborado proporcionando acceso a sus propiedades para poder realizar las recolectas en los distintos ambientes. Finalmente, se les agradece a los revisores anónimos cuyos comentarios ayudaron a mejorar este trabajo.

\section{RESUMEN}

Los macroinvertebrados acuáticos, y en especial los insectos, son el grupo de organismos más utilizado para determinar el estado de conservación de los ecosistemas acuáticos en estudios de calidad del agua y biomonitoreo acuático. La colección científica más importante de este grupo en el país se encuentra en el Museo de Zoología de la Universidad de Costa Rica y contiene material proveniente tanto de proyectos de investigación, tesis y trabajos de estudiantes como muestras recolectadas durante estudios y monitoreos ambientales realizados por consultores y diversas instituciones. En este trabajo se presenta un listado actualizado del material depositado, el cual incluye 98 familias y 341 taxones identificados (géneros, tribus o subfamilias) de once órdenes de insectos, siendo Coleoptera y Diptera los órdenes de mayor riqueza taxonómica. Se citan los trabajos que se han generado a partir de revisiones de la colección y los cuales incluyen registros nuevos de especies, géneros y de una familia para el país, así como las descripciones de especies nuevas y asociaciones de estadios inmaduros con sus adultos. Los principales desafíos 
para esta colección incluyen la identificación taxonómica de ciertos grupos, así como mantenerse al día con el constante crecimiento de la colección y la actualización de su base de datos.

Palabras clave: agua dulce; insectos acuáticos; macroinvertebrados; taxonomía; inventario.

\section{REFERENCIAS}

Adler, P. H., \& Currie, D. C. (2009). Simuliidae (black flies, bocones). In B. V. Brown, A. Borkent, J. M. Cumming, D. M. Wood, N. E. Woodley, \& M. A. Zumbado (Eds.), Manual of Central American Diptera (vol. I, pp. 389-406). Ottawa, Canada: NRC Research Press.

Bonada, N., Prat, N., Resh, V. H., \& Statzner, B. (2006). Developments in aquatic insect monitoring: a comparative analysis of recent approaches. Annual Review of Entomology 51(1), 495-523.

Brown, B. V., Borkent, A., Cumming, J. M., Wood, D. M., Woodley, N. E., \& Zumbado, M. A. (2009). Manual of Central American Diptera. Volumen I. Ottawa, Canada: NRC Research Press.

Chaverri, L. G. (2009). Culicidae (mosquitos, zancudos). In B. V. Brown, A. Borkent, J. M. Cumming, D. M. Wood, N. E. Woodley, \& M. A. Zumbado (Eds.), Manual of Central American Diptera (vol. I, pp. 369388). Ottawa, Canada: NRC Research Press.

De la Rosa, C. (2015). Chironomids: A personal journey. Chironomus Journal of Chironomidae Research 28, 30-35.

Drew, J. (2011). The role of natural history institutions and bioinformatics in conservation biology. Conservation Biology, 25(6), 1250-1252.

Figueiredo Moreira, F. F., Pacheco-Chaves, B., Springer, M., \& Da Rocha Silva Cordeiro, I. (2015). Two new Rhagovelia (Hemiptera: Heteroptera: Veliidae) from Costa Rica, with a key and new records from the country. Zootaxa, 3980(4), 477-500.

Flowers, R. E., (2010). Ephemeroptera. En M. Springer, A. Ramírez \& P. Hanson (Eds.), Macroinvertebrados de agua dulce de Costa Rica I. Revista de Biología Tropical, 58(Supl 4), 63-93.

Gutiérrez-Fonseca, P. E., (2010). Plecoptera. Macroinvertebrados de agua dulce de Costa Rica I. Revista de Biología Tropical, 58(Supl 4), 139-48.

Gutiérrez-Fonseca, P. E., \& Springer, M. (2011). Description of the final instar nymphs of seven species from Anacroneuria Klapalék (Plecoptera: Perlidae) in Costa Rica, and first record for an additional genus in Central America. Zootaxa, 2965, 16-38.
Gutiérrez-Fonseca, P. E., \& Springer, M. (2015). A new species of Anacroneuria Klapálek 1909 (Plecoptera: Perlidae) from Costa Rica and notes on the altitudinal distribution of the genus in Costa Rica. Zootaxa, 4058(4), 595-600.

Herrera, F. (2013a). Diversidad y distribución de los chinches Naucóridos (Hemiptera: Naucoridae) en Costa Rica (Tesis de Maestría). Sistema de Estudios Posgrado en Biología, Universidad de Costa Rica.

Herrera, F. (2013b). First record of the genus Ctenipocoris (Heteroptera: Naucoridae) in Central America, with a preliminary key to the American species and description of a new species. Zootaxa, 3731(3), 338-344.

Herrera, F., \& González, M. (2014). First description of macropterous male and female of Cryphocricos latus (Heteroptera: Naucoridae) Usinger. Journal of Natural History, 48, 51-55.

Herrera, F., \& Springer, M. (2012). First record of the Family Potamocoridae (Hemiptera: Heteroptera) in Costa Rica and of Coleopterocoris Hungerford, 1942 in Central America. Zootaxa, 3333, 66-68.

Herrera, F., \& Springer, M. (2014). New species of Potamocoris Hungerford (Heteroptera, Potamocoridae) from Costa Rica and a key to the species. Zootaxa, 3884(5), 492-496.

Hoeksema, B. W., van der Land, J., van der Meij, S. E. T., van Ofwegen, L. P., Reijnen, B. T., van Soest, R. W. M., \& de Voogd, N. J. (2011). Unforeseen importance of historical collections as baselines to determine biotic change of coral reefs: the Saba Bank case. Marine Ecology, 32, 135-141.

Kress, J. W. (2014). Valuing collections. Science, 346 (6215), 1310.

Lane, M. A. (1996). Roles of natural history collections. Annals of the Missouri Botanical Garden, 83, 536-545.

Pacheco-Chaves, B., DaRocha Silva Cordeiro, I., Figueiredo Moreira, F. F., \& Springer, M. (2018). The water striders (Hemiptera: Heteroptera: Gerridae) of Costa Rica: new species, checklist, and new records. Zootaxa 4471(3), 492-522.

Pacheco-Chaves, B., Figueiredo Moreira, F. F., \& Springer, M. (2014). New records of Gerromorpha (Insecta: Hemiptera: Heteroptera) from Costa Rica. Check List, 10(1), 180-186.

Padilla-Gil, D. N., \& Pacheco-Chaves, B. (2012). New records of Rheumatobates Bergroth (Hemiptera: Heteroptera: Gerridae) from the Pacific coast of Colombia and Costa Rica, with a key to males of Rheumatobates in the Eastern Tropical Pacific. Zootaxa, 3427, 33-46. 
Pinto, C. M., Baxter, B. D., Hanson, J. D., Mendez-Harclerode, F. M., Suchecki, J. R., Grijalva ... Bradley, R. D. (2010). Using museum collections to detect pathogens. Emerging Infectious Diseases, 16, 356-357.

Pyke, G. H., \& Ehrlich, P. R. (2010). Biological collections and ecological/environmental research: a review, some observations and a look to the future. Biological Reviews, 85, 247-266.

Ramírez, A. (2010). Odonata. Macroinvertebrados de agua dulce de Costa Rica I. Revista de Biología Tropical, 58(Supl 4), 97-136.

Ramírez, A., Altamiranda-Saavedra, M., Gutiérrez-Fonseca, P., \& Springer, M. (2011). The neotropical damselfly genus Cora: new larval descriptions and a comparative analysis of larvae of known species (Odonata: Polythoridae). International Journal of Odonatology, 14(3), 249-256.

Román-Heracleo, J., Springer, M., \& Ramírez, A. (2018) The larva of Perissolestes remotus (Williamson \& Williamson, 1924) (Zygoptera: Perilestidae). International Journal of Odonatology. DOI: 10.1080/13887890.2018.1511481

Rosenberg, D. M., \& Resh, V. H. (1993). Freshwater biomonitoring and benthic macroinvertebrates. New York, EEUU: Chapman \& Hall.

Specify Collections Consortium (2018). Specify 7. Lawrence, EEUU; Biodiversity Institute, University of Kansas. Retrieved from https://www.sustain.specifysoftware.org/products/specify-7/

Springer, M. (1998). Genera of aquatic insects from Costa Rica, deposited at the Museo de Zoología,
Universidad de Costa Rica. Revista de Biología Tropical, 46(Supl 6), 137-141.

Springer, M. (2006). Clave taxonómica para las larvas de las familias del orden Trichoptera (Insecta) de Costa Rica. Revista de Biología Tropical, 54(Supl 1), 273-286.

Springer, M. (2008). Aquatic insect diversity of Costa Rica: state of knowledge. Revista de Biología Tropical, 56(Supl 4), 273-295.

Springer, M. (2010). Trichoptera. Macroinvertebrados de agua dulce de Costa Rica I. Revista de Biología Tropical, 58(Supl 4), 151-198.

Springer, M., \& Acosta, R. (2003). First description of the larva of Pharceonus Spangler et Santiago-Fragoso, 1992, and new records for the genus (Coleoptera: Elmidae: Larainae). Aquatic Insects, 25(3), 219-223.

Springer, M., \& Bermúdez, J. (2018). Description of the larva and pupa of Limnephilus hamifer Flint, 1963 (Trichoptera, Limnephilidae) from Costa Rica. Zootaxa 4461(2), 277-285.

Springer, M., Echeverría, S., \& Gutiérrez, P. (2014). Costa Rica. In P. Alonso-EguíaLis, J. M. Mora, B. Campbell, \& M. Springer (eds.), Diversidad, conservación y uso de los macroinvertebrados dulceacuícolas de México, Centroamérica, Colombia, Cuba y Puerto Rico (pp. 119-155). Morelos, México: Instituto Mexicano de Tecnología del Agua, Jiutepec.

Suarez, A.V., \& Tsutsui, N. D. (2004). The value of museum collections for research and society. BioScience, 54(1), 66-74. 